\title{
Perspectives on Radiopharmaceutical Agents from the FDA
}

\author{
A Conversation between Lou Marzella, Johannes Czernin, and Thomas Hope
}

\author{
Lou Marzella ${ }^{1}$, Johannes Czernin ${ }^{2}$, and Thomas Hope ${ }^{3}$ \\ ${ }^{I}$ Food and Drug Administration, Silver Spring, Maryland; ${ }^{2}$ Department of Nuclear Medicine, UCLA School of Medicine, Los Angeles, \\ California; and ${ }^{3}$ Department of Radiology and Biomedical Imaging, University of California San Francisco, San Francisco, California
}

$\mathbf{J}$ ohannes Czernin, MD, editor in chief of The Journal of Nuclear Medicine, and Thomas Hope, MD, associate professor-inresidence at the University of California at San Francisco (UCSF), talked with Lou Marzella, MD, PhD, about his work in federal regulation and advancement of diagnostic and therapeutic radiopharmaceuticals. Dr. Marzella is the director of the Division of Imaging and Radiation Medicine (DIRM) in the Center for Drug Evaluation and Research (CDER) at the U.S. Food and Drug Administration (FDA) in Silver Spring, MD. The DIRM regulates imaging drugs, including contrast agents and radiopharmaceuticals, as well as therapeutic drugs for use in radiation injury.

Before joining the FDA, Dr. Marzella did research and taught at the University of Maryland School of Medicine in Baltimore. He trained at the University of Maryland, where he received his medical degree and completed a residency in family medicine, and he received a $\mathrm{PhD}$ in experimental pathology from the Karolinska Institutet in Stockholm, Sweden.

Dr. Czernin: Thank you for taking time out of your busy schedule to talk with us. You have led the DIRM for many years and overseen a significant increase in new-drug approvals. Can you tell us a little bit about your career and how it brought you to the FDA?

Dr. Marzella: I trained at the University of Maryland in Baltimore, and there I became interested in research and mechanisms of disease. I continued my basic research training in cell biology and pathology at the Karolinska Institute. In the 1980s, I joined the faculty of the University of Maryland School of Medicine to focus on research and teaching. What attracted me to the FDA was the clinical development within the Center for Biologics Evaluation and Research (CBER). There was tremendous excitement, with novel biotechnology products and new pharmacologic targets that were identified. At the CBER, I learned the art and the science of clinical trial design and analysis.

Dr. Czernin: So you came up through the drug approval process rather than radioactive drugs. Is that correct?

Dr. Marzella: Exactly. In 2003, the biotechnology products that I was reviewing were reassigned to the CDER. And so I joined the CDER. There I selected the Division of Medical Imaging. I didn't have experience in clinical imaging, as my clinical training was in family medicine. Our division is highly interdisciplinary. We have specialists in nuclear medicine, radiology, radiation oncology, and many more, and each of these specialties brings an understanding of

COPYRIGHT (C) 2021 by the Society of Nuclear Medicine and Molecular Imaging. DOI: 10.2967/jnumed.121.262450 the context of use for a drug. But the glue that ties us together is our understanding of clinical trials and scientific methodology. This is my expertise.

Dr. Hope: Radiopharmaceuticals are incredibly safe. We do many safety evaluations in new radiotracer development, including EKGs, blood tests, vital signs, etc. I'm not actually sure what we're looking for most of the time. How much of this is really required for drugs given at picomolar concentrations?

Dr. Marzella: It starts with the preclinical characterization. We have standards that we apply generally across drugs. These standards are not just FDA-specific or U.S.-specific; they are international standards. It's always a risk/benefit consideration. We appreciate the fact that the radiopharmaceuticals have no pharmacologic effects with very few exceptions and that there's a single or infrequent use. We have provided guidance to that effect. With regard to the clinical safety evaluations, we think that the basic characterization needs to be done in early studies. We typically require that there be several subjects studied for safety even above and beyond what's needed for efficacy. And we typically make allowances for the amount of data that must be obtained for products administered at microdose levels. We would like adverse events to be captured, including some follow-up after patient discharge to make sure that there are no late adverse events.

Dr. Czernin: A recent commentary about ${ }^{11}$ C-labeled compounds suggested that, because of their short half-lives and extremely favorable dosimetry, dosimetry requirements should be waived. It is important to recognize that academic research is notoriously underfunded and everything that can be removed from required procedures would be very helpful.

Dr. Hope: Let me also comment on that. Looking back 15 years, it was very difficult to get a radiopharmaceutical approved. In contrast, many radiopharmaceuticals were approved during the last 5 years because of enabling changes that the FDA has made. Can the process be further simplified?

Dr. Marzella: The legal expectation is that the radiation absorbed dose has to be calculated from preclinical and clinical data. The regulations require that first-in-humans studies of radiopharmaceuticals assess dosimetry. But I think that as the science 
advances and our experience increases, we need to see what data are not necessary. It is important to determine the biodistribution of the product. Therefore, dosimetry is almost a byproduct. We don't get any pushback from commercial manufacturers on this, because it's an important component of understanding drug pharmacokinetics and pharmacodynamics. But we do understand that the extrapolation from animals to humans is imperfect. We have undertaken a review of our experience with ${ }^{11} \mathrm{C},{ }^{18} \mathrm{~F}$, and ${ }^{68} \mathrm{Ga}$ to see to what extent preclinical studies could potentially be waived. I think that there is room for improvement here.

Dr. Czernin: I am hoping for a commonsense approach: let the market decide. If a product is clinically useless it will not be used.

Dr. Marzella: To be approved for marketing, products must show clinical utility. I think that, in a sense, imaging drugs do not need to show utility in terms of a beneficial effect on clinical outcome. If the administered product can produce an image to accurately diagnose disease, then that is a sufficient measure of utility. Also, single-arm intrapatient controlled studies are generally sufficient. For therapeutic isotopes it is much more complicated, in that you need to have parallel-arm, controlled, randomized studies.

Dr. Hope: Many of the imaging probes that we are studying in the United States today came out of places such as Germany, where initial analysis and development starts in a compassionateuse setting. This allows the quick evaluation of several imaging agents with low financial risk. A great example is prostate-specific membrane antigen (PSMA)-11. We do need safety and toxicity data. But I am not so sure whether we require it to get into the first 10 patients in order to see if the agent works. Is there a way you can think about getting probes into a small number of patients to see if the probe works? that that there must be a serious and unmet medical need. There has to be potential for the product to provide a significant advantage over what's available. The data from the clinical study need to be essential for developing the evidence required to market this product. And, of course, the trial should not be feasible without charging. Therefore, the first and the last conditions are relatively easy to meet. The "significant advantage" clause is the major hurdle. It's not supposed to be a "me too" drug. Making a product more broadly available is a significant advantage.

We encourage the role of the academic community in advanced product development. It's possible to get funded for the discovery part of new drugs. But then the challenge is how to move forward from there. So, what you guys have done for PSMA - the collaboration of 2 academic centers - is really the model that I hope will be further enhanced. We need more collaboration, more centers, more multicenter trials. And what needs to happen is that an infrastructure for such clinical trials is created. This collaboration could involve the funding agencies, the National Institutes of Health perhaps, or even drug companies.

Dr. Czernin: Once the FDA grants a new-drug application, the next part starts, which is insurance coverage. I think you told us once that there's an effort to establish more structured collaborations between the Centers for Medicaid and Medicare Services (CMS) and the FDA to streamline not only the new-drug approval process but the subsequent Medicare approval process. Can you comment on that?

Dr. Marzella: The 2 agencies established parallel review programs with the FDA and CMS, particularly for national coverage.

"FDA encourages the role of the academic community in advanced radiopharmaceutical product development."

Dr. Marzella: In practice, this is not the major obstacle for product development. The identification of new targets is very appealing and exciting. But the important work is really drudgery. You need to take the product through all the steps to demonstrate that it actually works. This requires scientific evidence. Imaging products are actually unique, in that the FDA and other agencies don't require clinical outcome data. You don't have to show that you're actually making a difference in patient management and patient outcomes as you do for other products. So, the clinical trial standards are already favorable.

Dr. Hope: In Europe the system appears to be set up to encourage quick evaluations, but the system is not set up to take them through phase 3 clinical trials toward approval.

Dr. Czernin: Our UCSF/UCLA PSMA studies were enabled by the FDA cost recovery mechanism, under which patients are, in fact, the study sponsors. They paid for the studies, for which we are extremely grateful. Without that, the diagnostic PSMA-11 trials would have been impossible. Are you expanding on the cost recovery opportunity?

Dr. Marzella: Yes, we would like to encourage that process, and we think that we have 2 different pathways. One is expanded access, where there are really minimal requirements for data collection. We like to reserve that approach for products for which substantial evidence exists. For cost recovery, the requirements are
Dr. Czernin: So, if academia had wanted to use this parallel approach of CMS and FDA review, we could have done that?

Dr. Marzella: Yes. But commercial manufacturers don't do this because the CMS requires clinical outcome data whereas the FDA does not. These clinical outcome-powered studies are expensive and carry substantial risks for the manufacturers. Third-party payers want to know what the product adds to patient management. The reason why manufacturers are not interested in the parallel review process is that the required trials are more complicated and more expensive. The FDA does not require comparative safety from a control arm and a treatment arm for diagnostic imaging studies.

Dr. Hope: Most radiopharmaceuticals are being developed to improve patient selection for potential therapy. What do we need to change to succeed in the next 5 years, particularly in the theranostics realm?

Dr. Marzella: Theranostics for use in oncology offers an opportunity for leveraging the development of diagnostics. So, one option would be to use a therapeutic trial to evaluate the potential for a diagnostic imaging test to select patients who have the pharmacologic target for treatment with the investigational drug. The advantages would be that you can treat everyone and you can determine whether the level of expression of the target accounts for response. My hope is that the theranostic approach will be 
applied in other therapeutic areas, for example, in neurodegenerative diseases where there's much progress in diagnostics. The relative lack of therapeutics impedes the progress of diagnostics.

Dr. Czernin: That's an important discussion about imaging biomarkers and their validation. Does the FDA have a structured approach for biomarker development?

Dr. Marzella: Both the European Medicines Agency and the FDA have pathways for qualification of imaging biomarkers. The problem is that to establish sensitivity and specificity relative to a reference standard might not be helpful if the value of the marker in clinical use is not established. Can you make an argument that an imaging marker affects patient management by complementing the yield of biopsies or by avoiding biopsies? Can it address whole-body heterogeneity of cancers? If so, that could be a productive way to develop imaging agents to see how they could affect patient management.

Dr. Hope: Switching gears here, can you comment on how COVID changed the way you have evaluated and reviewed applications? What are you learning from the experience?

Dr. Marzella: We had challenges in conducting inspections of clinical and manufacturing sites and had to minimize the risks to our personnel who travel to these sites. We had to develop more flexibility in terms of how we do inspections. Yes, we have substituted for inspections whenever possible. We have extended application times. We also changed the analysis plans of studies to introduce interim analyses because of problems with enrollment. The other obvious sign of adaptation is that the FDA has authorized products based on emergency-use authorizations without approval.

Dr. Czernin: Did the FDA, at least in the approval process for vaccines, experience a lot of political pressure for decisionmaking processes?
Dr. Marzella: Under emergency conditions there is a heightened interest in the FDA's actions on the part of manufacturers, various stakeholders, the public, and patient advocacy groups. It is not new for us that we have to be responsive to information requests. We interact with Congress and other government agencies in emergency situations. This public health emergency has put strains on the FDA in trying to identify the best ways to accommodate important needs. We also have been working hard to maintain public confidence in our public health mission.

The FDA is also involved in preparedness for national emergencies, whether from new infectious disease agents, nuclear disasters, or terrorist events. In addition, we need to continue to anticipate these dangers to public health. Our division is particularly involved in countermeasures for acute radiation emergencies. We just recently approved a drug against myelosuppression induced by acute radiation syndrome. This work is based on collaboration between the public sector and various levels of the government.

Dr. Hope: We have to come to the conclusion. I would like to thank you for your encouragement, support, and collaboration. The agency can help with bringing forward products that improve patient care. I really appreciate that you as an individual and your department have helped patients overall.

Dr. Marzella: I'd be interested in further discussions, maybe workshops. What can we do to promote drug development? If I may, I'd also like to advertise for experienced clinical investigators like you to join the FDA and work with us on developing new drugs.

Dr. Czernin: Thank you very much for your time and insights.

Dr. Marzella: Thank you. I appreciate the discussion and look forward to advancing the development of imaging and radiation medicine agents. Thank you very much for your work. 